\title{
Development and Validation of a Nomogram for Predicting the Probability of Adjacent Segmental Fractures After Vertebral Augmentation of Osteoporotic Vertebral Compression Fractures
}

\section{Qiujiang Li}

Ningxia Medical University

\section{Xingxia Long}

West China Hospital of Sichuan University

\section{Yinbin Wang}

Ningxia Hui Autonomous Region Peoples Hospital

\section{Xiaomin Fang}

Ningxia Hui Autonomous Region Peoples Hospital

\section{Donggeng Guo}

Ningxia Hui Autonomous Region Peoples Hospital

Jinhan Lv

Ningxia Hui Autonomous Region Peoples Hospital

Xuehua Hu

Ningxia Hui Autonomous Region Peoples Hospital

Lijun Cai ( $\sim$ scsiqj@163.com )

Ningxia Hui Autonomous Region Peoples Hospital

\section{Research Article}

Keywords: Osteoporosis vertebral compression fractures, vertebral augmentation, adjacent segmental fractures, nomogram

Posted Date: March 5th, 2021

DOl: https://doi.org/10.21203/rs.3.rs-263376/v1

License: (c) (1) This work is licensed under a Creative Commons Attribution 4.0 International License. Read Full License 


\title{
Development and validation of a nomogram for predicting the probability of
} adjacent segmental fractures after vertebral augmentation of osteoporotic vertebral compression fractures

Qiujiang Li ${ }^{1,2,+}$, Xingxia Long ${ }^{3,+}$, Yinbin Wang ${ }^{2}$, Xiaomin Fang ${ }^{2}$,

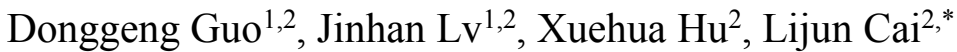

${ }^{1}$ Graduate School of Ningxia Medical University, Yinchuan, Ningxia, China.

${ }^{2}$ Department of Orthopedics,People's Hospital of Ningxia Hui Autonomous Region, Yinchuan, Ningxia, China.

${ }^{3}$ West China Hospital, Sichuan University,Sichuan,China

${ }^{*}$ Correspondence: Lijun Cai, No. 56, Zhengyuan Street, Yinchuan, Ningxia, 750002, China.

tQL and XL contributed equally to this manuscript

\begin{abstract}
Background

Adjacent segmental fractures are adverse events after vertebral augmentation of osteoporotic vertebral compression fractures(OVCFs). Predicting the risk of adjacent segmental fractures accurately after surgery is still a significant challenge for spinal surgeons. The aim of our study was to identify predictive factors of the risk of adjacent segmental fractures after vertebral augmentation of OVCFs and develop a nomogram.
\end{abstract}

\section{Methods}

A nomogram was compiled based on the training cohort of 403 patients, who were hospitalized for OVCFs and performed vertebral augmentation, in the People's Hospital of Ningxia Hui Autonomous Region from June 2014 to December 2016. The independent predictive factors of postoperative adjacent segmental fractures were determined by LASSO, univariate analysis and multivariate logistic regression analysis. Then, establish a nomogram based on these independent predictors. We assessed nomograme using variety methods, including area under the curve (AUC), calibration curve and decision curve analysis (DCA).The above results were verified 
by the validation cohort of 159 patients, who were hospitalized for OVCFs and performed vertebral augmentation between January 2017 and June 2018.

\section{Results}

The establishment of the nomogram was based on six independent predictors, were determined by multivariate analysis, including age, bone cement injection, bone cement leakage, contact between bone cement and vertebral endplates, bone cement dispersion, and anti-osteoporosis treatment. In the training and validation cohort, the AUC of the nomogram were 0.882 (95\% confidence interval, $0.824-0.940)$ and 0.869 (95\% confidence interval: $0.811-0.927)$,respectively.In the training and validation cohort, the optimal calibration curves demonstrated the coincidence between prediction and actual status, and the decision curve analysis demonstrated that the full model had the highest clinical net benefit across the entire range of threshold probabilities.

\section{Conclusion}

The risk prediction model shows a satisfactory prediction effect and could quantify the probability of adjacent segmental fractures after vertebral augmentation of OVCFs.

\section{Key words}

Osteoporosis vertebral compression fractures; vertebral augmentation; adjacent segmental fractures; nomogram

\section{Background}

Osteoporotic vertebral compression fractures(OVCFs) mainly cause sudden severe pain, progressive kyphosis, decreased quality of life, and increased mortality ${ }^{[1-3]}$. Although patients with milder conditions can be treated with bed rest and pain medications, many patients cannot receive non-surgical treatment due to additional health problems caused by immobilization ${ }^{[4]}$. As the most commonly used minimally

invasive surgical methods, percutaneous vertebroplasty (VP) and percutaneous balloon kyphoplasty (BKP) have been accepted for the treatment of OVCFs in the past decades ${ }^{[5,6]}$. This operation inserts a large hollow needle into the vertebral body 
percutaneously through the pedicle, and injects polymethylmethacrylate (PMMA) to stabilize the vertebral body, prevent further collapse, and achieve rapid pain relief and functional recovery ${ }^{[7-9]}$.

However, with the improvement and popularization of vertebroplasty technology, there are more and more related complications. Among them, postoperative adjacent segmental fractures are the most common, which will reduce the quality of life of patients and bring more economic burden to society ${ }^{[10,}{ }^{11]}$. By calculating the absolute risk of adjacent vertebral fractures after vertebroplasty in patients with osteoporotic vertebral compression fractures, preventive and therapeutic interventions can be provided to high-risk groups, and low-risk groups can be avoided unnecessary Of care. Therefore, it is necessary for us to identify high-risk patients early and take special intervention measures to reduce the risk of adjacent vertebral fractures after vertebroplasty.

New predictive models, including lasso regression and nomogram based on multiple logistic regression, have been developed and validated to predict postoperative vertebral compression fractures ${ }^{\left[12,{ }^{13]}\right.}$. As far as we know, this is the first attempt to develop a nomogram-based method for adjacent vertebral fractures after vertebroplasty. Our results provide a new perspective for adjacent vertebral fractures after surgery, and have strong significance for clinical applications. To improve clinical decision-making, doctors need to know the risk of vertebral compression fractures after vertebroplasty. Therefore, this study will develop, verify, and evaluate the clinical applicability of the risk prediction model for adjacent vertebral fractures after vertebroplasty in patients with osteoporotic vertebral compression fractures.

\section{Patients and methods}

\section{patient}

From June 2014 to June 2018, a total of 892 patients in our institution were diagnosed with osteoporotic vertebral compression fractures. This study screened a total of 562 patients who met the criteria. From June 2014 to December 2016, 403 patients were included in the training cohort. In addition, a validation cohort of 159 
patients from January 2017 to June 2018 was recruited, with the same standards as the training cohort. In the training cohort, 49 patients had postoperative vertebral compression fractures, and 354 patients had no postoperative vertebral compression fractures. The inclusion criteria are as follows: (1) bone mineral density $\mathrm{T}$ value $<-2.5 \mathrm{SD}$; (2) fractured vertebral body conforms to the imaging characteristics, and the pain is obvious (VAS > 6 ). The exclusion criteria are as follows: (1) Space-occupying lesions of the spinal canal; (2) Kummell's Disease and other delayed fractures and nonunion diseases; (3) Abnormal increase in blood inflammatory indexes, suggesting infection. All methods are carried out in accordance with relevant guidelines and regulations. This retrospective study was approved by the ethics committee of the People's Hospital of Ningxia Hui Autonomous Region (the Third Clinical College of Ningxia Medical University). Informed consent of all subjects was obtained.

\section{Surgical methods}

Prone hyperextension position, fluoroscopy, mark the pedicle of the fractured vertebral body. After local anesthesia, puncture through the pedicle, fluoroscopy lateral radiographs show that a working channel is established when the needle tip crosses the midline of the vertebral body. After the prepared bone cement is drawn into the wire shape, it is slowly injected into the vertebral body. The whole process is completed with the help of C-arm X-ray machine to avoid bone cement leakage. Each patients take regular anti-osteoporosis treatment (oral calcium and vitamin D) after surgery. If some patients have no fever or other discomfort, they will receive intravenous infusion of zoledronic acid (Aclasta, $5 \mathrm{mg} / 100 \mathrm{ml}$ ) on the first day after surgery. Frequency of medication Once a year for 3 years.

\section{Observation indicators}

(1) General information: gender, ethnicity, age, body mass index (BMI), number of fractured vertebral bodies, and fractured vertebral body segments. (2) Surgical factors: surgical method (PVP/PKP), average bone cement dosage, bone cement leakage, bone cement dispersion, contact between bone cement and endplate, available anti-osteoporotic treatment. (3) Past Medical History: fracture, diabetes, hypertension,etc. Good diffusion of bone cement is defined as: the bone cement has 
crossed the midline of the vertebral body no matter anteroposterior and lateral radiographs. Available anti - osteoporotic treatment is defined as oral calcium and vitamin D, infusion zoledronic acid via intravenous annual.

\section{Statistical Analysis}

Continuous variables are expressed by the mean standard deviation. If they are normally distributed, the $t$ test is used for analysis. Otherwise, the data is expressed by the median and interquartile range, and the Mann-Whitney $U$ test is used. Categorical variables are expressed in frequency and percentage, and analyzed using Chi-square test or Fisher's exact test. The texture feature selection using the minimum absolute shrinkage and selection operator (lasso) regression model is used for data dimensionality reduction and feature selection. The variables with $\mathrm{P}<0.05$ in the univariate analysis were subjected to multivariate logistic regression analysis, and the variables with $\mathrm{P}<0.05$ were considered as possible predictors. Based on multi-factor logistic regression analysis, a predictive nomogram was established in the training queue. In order to further evaluate the resolving power of the nomogram, the area under the receiver operating characteristic curve was measured. A calibration curve was generated to evaluate the calibration of the non-adhesive nomogram. After that, the nomogram constructed in the training queue was further verified in the verification queue. Using the same method as above, evaluate the model's identification and calibration performance in the validation cohort. Finally, we assessed whether the model improves forecasted net income through decision curve analysis (DCA). A P value of 0.05 is considered statistically significant. We used SPSS version 24(IBM Corporation, Armonk, NY, USA) and R version 3.6.1(R Foundation for Statistical Computing, Vienna, Austria) for data analysis.

\section{Results}

\section{Clinical features}

There were a total of 403 patients in the training cohort, of which 49 patients $(12.16 \%)$ had secondary vertebral compression fractures 2 years after surgery. In the verification cohort, there were a total of 159 patients. There were 17 patients $(10.69 \%)$ 
of compression fractures (Table 1). The two groups were mostly elderly patients with an average age of more than 80 years old. The fractured vertebral bodies were located in the T10 L2 segment. The average amount of bone cement injected into each fractured vertebral body was $(4.02 \pm 1.13,4.43 \pm 0.88 \mathrm{ml})$ (Table 1) ). Despite the time difference, the baseline features between the two groups are comparable, indicating that they are suitable for use as training and validation data sets.

\begin{tabular}{|c|c|c|c|c|c|c|}
\hline & \multicolumn{3}{|c|}{ Training cohort $(n=403)$} & \multicolumn{3}{|c|}{ Validation cohort $(n=159)$} \\
\hline & $\operatorname{NRF}(n=354)$ & $\operatorname{RF}(n=49)$ & $\mathbf{P}$ & $\operatorname{NRF}(n=142)$ & $\operatorname{RF}(n=17)$ & $\mathbf{P}$ \\
\hline \multicolumn{7}{|l|}{ Gender,n(\%) } \\
\hline Male & $61(17.23 \%)$ & $10(20.41 \%)$ & 0.584 & $39(27.46 \%)$ & $4(23.53 \%)$ & 0.730 \\
\hline Female & $293(82.77 \%)$ & $39(79.59 \%)$ & & $103(72.54 \%)$ & $13(76.47 \%)$ & \\
\hline \multicolumn{7}{|l|}{ Age,years,n(\%) } \\
\hline$<60$ & $22(6.21 \%)$ & $1(2.04 \%)$ & 0.003 & $6(4.23 \%)$ & $1(5.88 \%)$ & 0.006 \\
\hline $60 \sim 70$ & $140(39.55 \%)$ & $12(24.49 \%)$ & & $62(43.66 \%)$ & $3(17.65 \%)$ & \\
\hline $70 \sim 80$ & $144(40.68 \%)$ & $20(40.82 \%)$ & & $54(38.03 \%)$ & $5(29.41 \%)$ & \\
\hline$>80$ & $48(13.56 \%)$ & $16(32.65 \%)$ & & $20(14.08 \%)$ & $8(47.06 \%)$ & \\
\hline \multicolumn{7}{|l|}{ Population,n(\%) } \\
\hline Han & $316(89.27 \%)$ & $46(93.88 \%)$ & 0.317 & $124(87.32 \%)$ & $13(76.47 \%)$ & 0.221 \\
\hline Hui & $38(10.73 \%)$ & $3(6.12 \%)$ & & $18(12.68 \%)$ & $4(23.53 \%)$ & \\
\hline BMI $\left(\mathrm{kg} / \mathrm{m}^{2}\right)$ & $23.82 \pm 3.55$ & $23.14 \pm 3.36$ & 0.208 & $23.21 \pm 3.86$ & $24.13 \pm 3.38$ & 0.351 \\
\hline \multicolumn{7}{|l|}{ Diabetes,n(\%) } \\
\hline No & $320(90.40 \%)$ & $46(93.88 \%)$ & 0.429 & $130(91.55 \%)$ & $15(88.24 \%)$ & 0.649 \\
\hline Yes & $34(9.60 \%)$ & $3(6.12 \%)$ & & $12(8.45 \%)$ & $2(11.76 \%)$ & \\
\hline \multicolumn{7}{|l|}{ Hypertension,n(\%) } \\
\hline No & $195(55.08 \%)$ & $23(46.94 \%)$ & 0.284 & $81(57.04 \%)$ & $11(64.71 \%)$ & 0.545 \\
\hline Yes & $159(44.92 \%)$ & $26(53.06 \%)$ & & $61(42.96 \%)$ & $6(35.29 \%)$ & \\
\hline \multicolumn{7}{|l|}{ Fractures,n(\%) } \\
\hline No & $309(87.29 \%)$ & $36(73.47 \%)$ & 0.010 & $133(93.66 \%)$ & $16(94.12 \%)$ & 0.942 \\
\hline Yes & $45(12.71 \%)$ & $13(26.53 \%)$ & & $9(6.34 \%)$ & $1(5.88 \%)$ & \\
\hline \multicolumn{7}{|c|}{ Augmentation segment,n(\%) } \\
\hline T4-T9 & $35(9.89 \%)$ & $2(4.08 \%)$ & 0.065 & $17(11.97 \%)$ & 0 & 0.307 \\
\hline T10-L2 & $192(54.24 \%)$ & $35(71.43 \%)$ & & $85(59.86 \%)$ & $11(64.71 \%)$ & \\
\hline L3-L5 & $127(35.88 \%)$ & $12(24.49 \%)$ & & $40(28.17 \%)$ & $6(35.29 \%)$ & \\
\hline Surgical method,n(\%) & & & 0.396 & & & 0.325 \\
\hline PVP & $194(54.80 \%)$ & $30(61.22 \%)$ & & $74(52.11 \%)$ & $11(64.71 \%)$ & \\
\hline PKP & $160(45.20 \%)$ & $19(38.78 \%)$ & & $68(47.89 \%)$ & $6(35.30 \%)$ & \\
\hline Bone cement dosage, $\mathrm{ml}$ & $4.02 \pm 1.13$ & $4.43 \pm 0.88$ & 0.014 & $3.81 \pm 0.87$ & $4.56 \pm 0.77$ & 0.001 \\
\hline
\end{tabular}




\section{Bone cement leakage,n(\%)}

$\begin{array}{llllllll}\text { No } & 238(67.23 \%) & 20(40.82 \%) & <0.001 & 97(68.31 \%) & 5(29.41 \%) & 0.002 \\ \text { Yes } & 116(32.77 \%) & 29(59.18 \%) & 45(31.69 \%) & 12(70.59 \%) & \\ \text { Bone cement dispersion, n(\%) } & & & & & & \\ \text { No } & 102(28.81 \%) & 33(67.35 \%) & <0.001 & 38(26.76 \%) & 9(52.94 \%) & 0.025 \\ \text { Yes } & 252(71.19 \%) & 16(32.65 \%) & 104(73.24 \%) & 8(47.06 \%)\end{array}$

Contact between bone cement and endplate,n(\%)

No

$53(14.97 \%) \quad 28(57.14 \%)<0.001 \quad 24(16.90 \%) \quad 5(29.41 \%) \quad 0.207$

Yes

$301(85.03 \%) 21(42.86 \%) \quad 118(83.10 \%) \quad 12(70.59 \%)$

Available anti-osteoporotic

treatment,n(\%)

\begin{tabular}{lccccc} 
No & $95(26.84 \%)$ & $36(73.47 \%)$ & $<0.001$ & $40(28.17 \%)$ & $13(76.47 \%)<0.001$ \\
Yes & $259(73.16 \%)$ & $13(26.53 \%)$ & $102(71.83 \%)$ & $4(23.53 \%)$ \\
\hline
\end{tabular}

Table 1. Demographics and clinical characteristics of study subjects. NRF: no adjacent segmental fractures; RF: adjacent segmental fractures

\section{Feature selection and independent risk factors of postoperative vertebral compression fractures}

Among the texture features (Figure 1A, B), based on the 403 patients in the training cohort, 17 features were reduced to 6 potential predictors, which showed non-zero coefficients in the lasso logistic regression model. Table 1 summarizes the demographics, clinical characteristics, and imaging data of subjects in the recompression and non-recompression vertebral fracture groups after vertebral augmentation. Table 1 lists all the predictor variables, evaluated by univariate and multivariate logistic regression analysis. Between recompression and non-recompression fracture group, there are differences in age, bone cement injection, bone cement leakage, bone cement dispersion, contact between bone cement and endplate, and whether anti-osteoporosis treatment is available. At the same time, the above variables have also been identified as independent risk factors for adjacent segmental fractures. In addition, Table 2 shows the intercept, $\beta$ coefficient and odds ratio of the multivariate logistic regression analysis. 

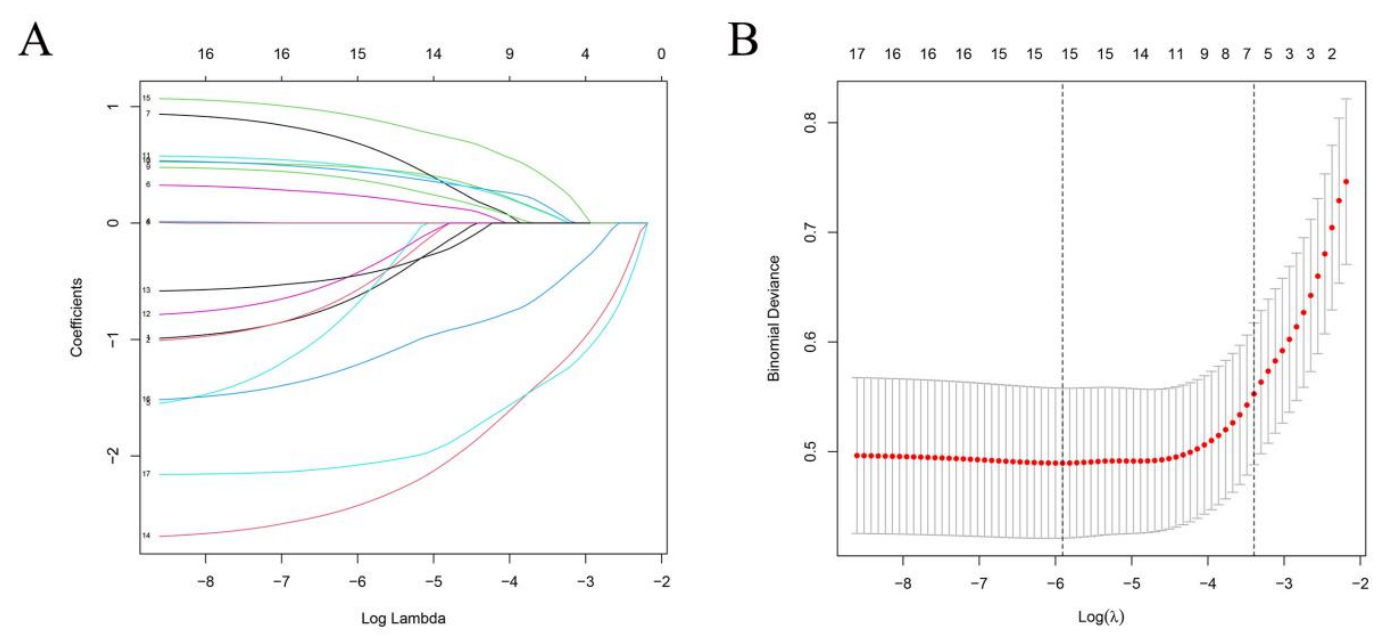

Figure 1 Demographic and clinical feature selection using LASSO binary logistic regression model. Note: 17 features of A cable coefficient profile. According to the logarithmic $(\lambda)$ sequence to draw the coefficient distribution map. Five-fold cross-validation is used to draw vertical lines at the selected values, where the best $\lambda$ results in six features with non-zero coefficients.The best parameter $(\lambda)$ in the B lasso model is selected through the minimum criterion using five-fold cross validation. The relationship between the partial likelihood deviation (binomial deviation) curve and the $\operatorname{logarithm}(\lambda)$ is drawn.

Abbreviations: LASSO, minimum absolute shrinkage, selection operator; SE, standard error.

\begin{tabular}{|c|c|c|c|c|c|}
\hline & B & SE & Wald & $\mathbf{P}$ & OR(95\%CI) \\
\hline Age & & & 5.671 & 0.129 & \\
\hline $60 \sim 70$ vs $<60$ & -2.439 & 1.261 & 3.74 & 0.043 & $0.087(0.007 \sim 1.033)$ \\
\hline $70 \sim 80$ vs $<60$ & -1.016 & 0.571 & 3.16 & 0.075 & $0.362(0.118 \sim 1.11)$ \\
\hline$>80$ vs $<60$ & -0.718 & 0.518 & 1.925 & 0.165 & $0.488(0.177 \sim 1.345)$ \\
\hline Fractures（yes） & -0.743 & 0.546 & 1.851 & 0.174 & $0.476(0.163 \sim 1.387)$ \\
\hline Bone cement dosage & & & 8.801 & 0.032 & \\
\hline $3 \sim 4 \mathrm{ml}$ vs $<3 \mathrm{ml}$ & -0.99 & 0.541 & 3.355 & 0.067 & $0.371(0.129 \sim 1.072)$ \\
\hline $4 \sim 4.5 \mathrm{ml} \mathrm{vs}<3 \mathrm{ml}$ & -1.145 & 0.492 & 5.402 & 0.02 & $0.318(0.121 \sim 0.836)$ \\
\hline
\end{tabular}




\begin{tabular}{lccccc}
\multicolumn{1}{c}{$>4.5 \mathrm{ml}$ vs $<3 \mathrm{ml}$} & -2.01 & 0.886 & 5.143 & 0.023 & $0.134(0.024 \sim 0.761)$ \\
Anti-osteoporosis treatment (yes) & 2.303 & 0.465 & 24.488 & $<0.001$ & $10.009(4.019 \sim 24.923)$ \\
Bone cement leakage (yes) & -1.093 & 0.423 & 6.671 & 0.01 & $0.335(0.146 \sim 0.768)$ \\
Bone cement dispersion (yes) & 0.799 & 0.433 & 3.401 & 0.065 & $2.223(0.951 \sim 5.195)$ \\
Contact with endplate (yes) & 2.275 & 0.469 & 23.533 & 0 & $9.729(3.88 \sim 24.394)$ \\
Augmentation segment & & & 1.822 & 0.402 & \\
$\quad$ T10-L2 vs T4 T9 & -0.915 & 0.991 & 0.854 & 0.356 & $0.400(0.057 \sim 2.791)$ \\
$\quad$ L3 L5 vs T4 T9 & 0.314 & 0.442 & 0.502 & 0.478 & $1.368(0.575 \sim 3.257)$ \\
Constants & -1.637 & 0.948 & 2.979 & 0.084 & \\
\hline
\end{tabular}

Table 2. Multivariate analysis in training cohort.

\section{Draw a nomogram through the training queue}

The model was established by multivariate logistic regression analysis to obtain independent risk factors (Figure 2). Among them, seven factors were used: age, bone cement injection volume, bone cement leakage, bone cement dispersion, contact between bone cement and endplate, and available anti - osteoporotic treatment. Each predictor is located on the relevant axis, and a straight line is drawn to the vertex axis to obtain a point based on the predictor. The total score is calculated by adding all the scores obtained from each predictor. The final sum is placed on the total points axis, and a straight line is drawn from there to get the non-fixed probability.

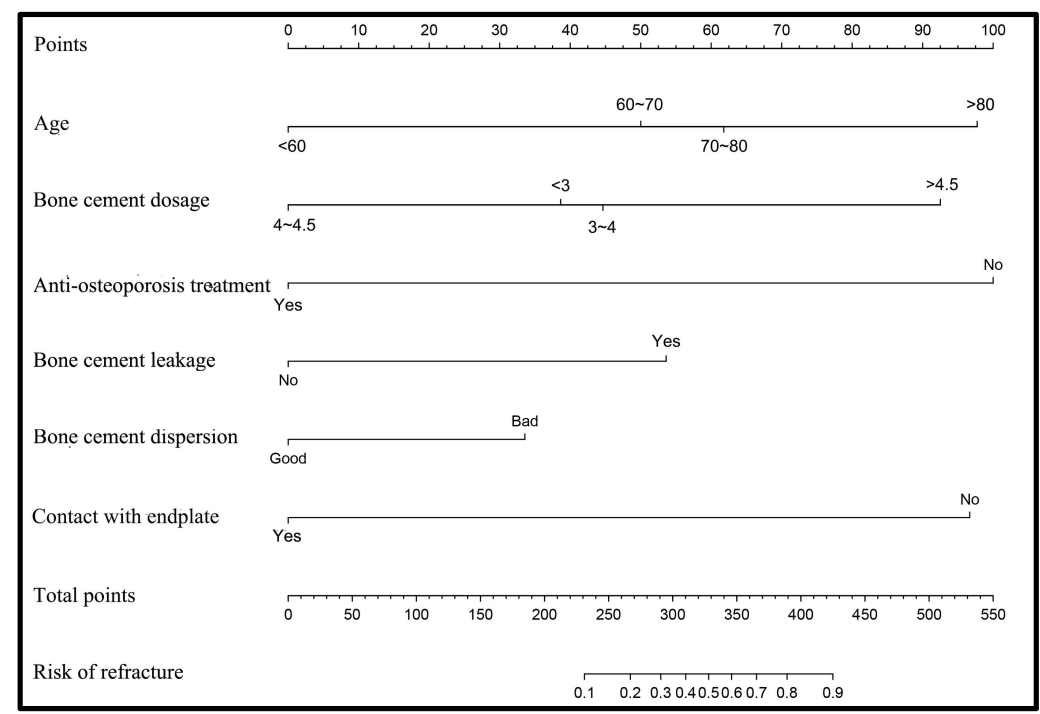

Figure 2. The developed nomogram of adjacent segmental fractures after vertebral 
augmentation.

Note: The nomogram of postoperative vertebral compression fractures was developed in the cohort, including age, bone cement injection volume, bone cement leakage, bone cement dispersion, contact between bone cement and endplate, and available anti - osteoporotic treatment.

\section{Verification of nomogram}

In the training cohort (Figure 3A), ROC showed that the obtained model has a fairly good discrimination ability, with an AUC of 0.882 (95\% confidence interval, 0.824-0.940), indicating that it is accurate to predict the risk of adjacent segmental fractures after vertebral augmentation. The calibration curve shows that the prediction of the nomogram is highly consistent with the actual observation (Figure 3B). In the validation cohort, the AUC of the model was 0.869 (95\% confidence interval: 0.811-0.927) (Figure 3D). The same calibration curve shows the prediction and observation of the probability of adjacent segmental fractures after vertebral augmentation (Figure 3E).

\section{DCA curve analysis}

In the training cohort, DCA showed that if the threshold probability of patients and doctors is greater than $2 \%$ and less than $96 \%$, using the nomogram to predict the risk of postoperative vertebral compression fractures is more beneficial than the program (Figure 3C).Similarly, in the validation cohort, DCA showed that if the threshold probability of patients and doctors is greater than $2 \%$ and less than $84 \%$, using nomogram to predict postoperative adjacent segmental fractures has a net benefit (Figure 3F). In general, the nomogram is feasible and can be used to make reasonable predictions. 


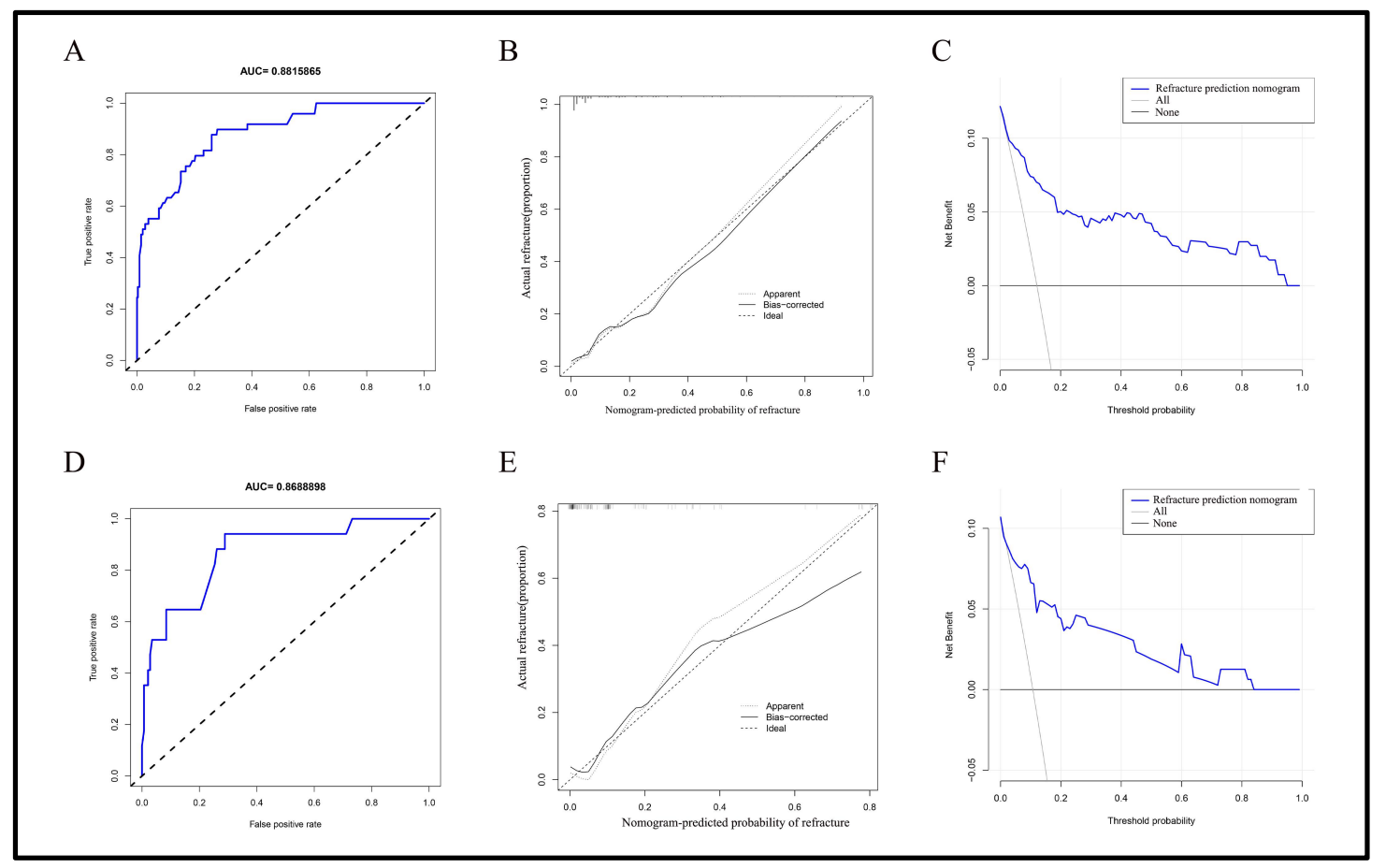

Figure 3. The receiver operating characteristic curve (A), calibration curve (B) and decision curve analysis $(\mathrm{C})$ of the training cohort. Receiver operating characteristic curve (D), calibration curve (E) and decision curve analysis $(F)$ of the validation cohort.

\section{Discuss}

In recent years, the nomogram model has been widely used in oncology and imaging omics research ${ }^{[14-16]}$. The nomogram has a good digital interface, more accurate and easier to understand predictive ability, which can better help clinical decision-making ${ }^{[15]}$.We applied this nomogram for the first time to the prediction of adjacent segmental fractures after vertebral augmentation of osteoporotic vertebral compression fractures. This study integrates the risk factors of demographics, surgery, and treatment characteristics into an easy-to-use nomogram, using only six easily available variables to predict the risk of adjacent segmental fractures after vertebral augmentation. This study provides patients of OVCFs with a relatively accurate prediction tool for postoperative adjacent segmental fractures. At the same time, the external verification results of this study also show good identification and calibration capabilities, especially the high AUC results of the model show that the nomogram 
can be widely and accurately applied to large sample studies.

In our study, six independent variables were identified as predictors of postoperative vertebral compression fractures, and the relationship had been widely reported in previous studies ${ }^{[17]}$. However, there are many reports on the influencing factors and incidence of adjacent segmental fractures after vertebral augmentation ${ }^{[18,19]}$. The reasons for the significant difference in morbidity are related to the inclusion criteria of OVCFs, surgical skills, postoperative anti-osteoporosis treatment and other factors, and may also be related to the medical level of different countries and the incidence of osteoporosis ${ }^{[20,21]}$. However, it is difficult to achieve homogeneity of research in this area, so there is no higher quality research report. In this study, 49 of the 403 patients had secondary vertebral fractures, and the incidence was $12.16 \%$. This result is similar to that reported by Zhang ${ }^{[22]}$.

At present, the pathogenesis of adjacent segmental fractures after vertebral augmentation is still unclear, but most scholars agree that it is related to the natural course of osteoporosis and the factors related to vertebral augmentation surgery ${ }^{[18]}$. In terms of age, the age of the recompression fractured group was significantly higher than the non-recompression fractured group, and multivariate logistic analysis found that age was an independent risk factor for adjacent segmental fractures after vertebral augmentation. As the age increased, postoperative the risk of recompression fracture is also significantly increased ${ }^{[23-25]}$. Regardless of whether patients with osteoporosis are combined with trauma, if regular and systematic anti-osteoporosis treatment is not given after the first vertebral fracture, more than $20 \%$ of patients will have vertebral fractures again. And systematic anti-osteoporosis treatment can slow down the process of osteoporosis and reduce the occurrence of recompression fractures ${ }^{[26,27]}$. Bawa et al ${ }^{[28]}$ conducted a 3-year follow-up study of 31,069 OVCFs patients and showed that anti-osteoporosis treatment can significantly reduce the incidence of fractures, and the risk of recompression fracture within 3 years can be reduced by about $40 \%$. Multivariate analysis in this study found that available anti - osteoporotic treatment is an important risk factor for adjacent segmental fractures after PVP/PKP. 
Therefore, anti - osteoporotic treatment should be a routine treatment in patients with OVCFs undergoing vertebral augmentation to reduce the risk of adjacent segmental fractures.

Kwon et al. ${ }^{[29]}$ reported that it is necessary to inject as much bone cement as possible for treatment, and the effect is best when the injected amount reaches $27.8 \%$ of the volume of the fractured vertebral. Additionally, other studies found that the amount of bone cement injected is positively related to the relief of postoperative pain, so they recommend using the largest volume of bone cement ${ }^{[30,31]}$. However, the above study did not consider long-term results and adjacent segmental fractures during follow-up. Excessive bone cement injection can increase the strength of the vertebral and at the same time cause an increase in the risk of adjacent segmental fractures and bone cement leakage. Many studies have shown that the pressure of the intervertebral disc can cause leakage of the cement and may cause the endplate of the adjacent vertebral to deflect, leading to be fractured. Zhu et al. ${ }^{[32]}$ found that the amount of bone cement in the thoracic should be less than $3.5 \mathrm{ml}$, and the lumbar should not exceed 4 $\mathrm{ml}$, which can effectively avoid bone cement leakage to a certain extent. The results of this study show that too much $(>4.5)$ and too little $(<4 \mathrm{ml})$ of bone cement injection will affect the occurrence of postoperative vertebral fractures. When the amount of bone cement injection is controlled at $4 \sim 4.5 \mathrm{ml}$, the postoperative follow-up the risk of vertebral fractures is the lowest. Although the results of this study confirm that proper amount of bone cement injection can effectively reduce the risk of postoperative fractures, individualized treatment should be implemented for patients with different compression degrees or vertebral body injuries. The amount of bone cement injection must be optimized clinically. Infusion improves clinical efficacy.

The results of this study also found that poor dispersion of bone cement in the vertebral body and failure to contact the upper and lower endplates are risk factors for postoperative adjacent segmental fractures. A biomechanical study by Chevalier ${ }^{[33]}$ reported that the balloon expansion effect of vertebral augmentation squeezes the loose cancellous bone to the surroundings to form a cavity, causing the injected bone cement to be blocked by the surrounding dense trabecular bone, reducing dispersion 
of bone cement. Studies have used X-rays to observe whether the bone cement crosses the midline to evaluate the diffusion of bone cement in the vertebral body ${ }^{[34]}$. It is found that the clinical effect of bone cement crossing the midline of the vertebral body is better than that of bone cement on one side, and the risk of bone cement leakage is low. When the bone cement is sufficiently diffused to contact the upper and lower endplates, the load on the vertebral body can be transferred in an orderly and uniform manner in the order of the upper endplate, bone cement, and lower endplate, which greatly enhances the strength and rigidity; On the contrary, when the bone cement only touches one endplate, the increase rate of vertebral body strength and stiffness is significantly reduced. Therefore, full consideration of the dispersion of bone cement and contact with the upper and lower endplates can reduce the risk of recopmression fractures to a certain extent.

This study established a nomogram model based on a large cohort, and successfully verified it in an independent cohort. All variables included in the nomogram are easy to determine. By calculating the points for each of the six variables, the spine surgeon can easily estimate the risk of recopmression fractures after surgery. Based on the evaluation results, preoperative management strategies can be specified to reduce the risk of recopmression fractures in high-risk patients. Similarly, for low-risk patients, some preventive measures can be reduced to reduce the economic burden and the risk of side effects.

Our research also has some limitations. First, this is a retrospective study, so there may be inherent selection bias. However, we included as many preoperative and surgical factors as possible on the basis of a large sample to minimize deviation. Second, although this nomogram has been verified by an independent cohort, we must realize that the incidence of postoperative vertebral fractures reported by different hospitals, regions and countries is different, which may limit this nomogram to a small number of Hospital application. Third, because some data was lost in the retrospective study, the bone mineral density was not considered in the multivariate regression analysis. The sensitivity and specificity of nomogram can be further improved through multi-center retrospective validation studies or prospective 
randomized clinical trials, which will provide high-level evidence for future clinical applications.

\section{Conclusion}

This study found that advanced age, bone cement injection volume $(<4$ or $>4.5 \mathrm{ml}$ ), bone cement leakage, poor bone cement dispersion, bone cement not contacting the endplate, and available anti - osteoporotic treatment are the independent risk factors of adjacent segmental fractures after vertebral augmentation for osteoporotic vertebral compression fractures. The nomogram containing the above six predictors can accurately predict the risk of adjacent segmental fractures after surgery.

\section{Disclosure}

The authors have no personal, financial, or institutional interests in any of the drugs, materials, or devices described in this article.

\section{Funding}

This study was funded by Ningxia Hui Autonomous Region Science and Technology Benefiting People Special Project(2018KJHM00) .

\section{Availability of data and materials}

Please contact the corresponding author for data requests.

\section{Author details}

${ }^{1}$ Graduate School of Ningxia Medical University, Yinchuan, Ningxia, China.

${ }^{2}$ Department of Orthopedics,People's Hospital of Ningxia Hui Autonomous Region, Yinchuan, Ningxia, China.

${ }^{3}$ West China Hospital, Sichuan University,Sichuan,China

*Correspondence: Lijun Cai, No. 56, Zhengyuan Street, Yinchuan, Ningxia, 750002, China.

\section{Reference}

[1] Firanescu C E, de Vries J, Lodder P, et al. Vertebroplasty versus sham procedure for painful acute osteoporotic vertebral compression fractures (VERTOS IV): 
randomised sham controlled clinical trial[J]. BMJ,2018,361:k1551.

[2] Luthman S, Widen J, Borgstrom F. Appropriateness criteria for treatment of osteoporotic vertebral compression fractures[J]. Osteoporos Int,2018,29(4):793-804.

[3] Svensson H K, Olsson L E, Hansson T, et al. The effects of person-centered or other supportive interventions in older women with osteoporotic vertebral compression fractures-a systematic review of the literature[J]. Osteoporos Int,2017,28(9):2521-2540.

[4] Zhao G, Liu X, Li F. Balloon kyphoplasty versus percutaneous vertebroplasty for treatment of osteoporotic vertebral compression fractures (OVCFs) $[\mathrm{J}]$. Osteoporos Int,2016,27(9):2823-2834.

[5] Xu J C, Wu G H, Zhou L L, et al. Two unilateral puncturation comparative analyses of multiple-level fresh osteoporotic vertebral body compression fractures treated with percutaneous vertebroplasty guided by $\mathrm{C}$-arm fluoroscopy or in senile patients[J]. Eur Rev Med Pharmacol Sci,2017,21(7):1456-1461.

[6] Buchbinder R, Johnston R V, Rischin K J, et al. Percutaneous vertebroplasty for osteoporotic vertebral compression fracture[J]. Cochrane Database Syst Rev,2018,11:CD006349.

[7] He Z, Zhai Q, Hu M, et al. Bone cements for percutaneous vertebroplasty and balloon kyphoplasty: Current status and future developments[J]. J Orthop Translat,2015,3(1):1-11.

[8] Yang C C, Chien J T, Tsai T Y, et al. Earlier Vertebroplasty for Osteoporotic Thoracolumbar Compression Fracture May Minimize the Subsequent Development of Adjacent Fractures: A Retrospective Study[J]. Pain Physician,2018,21(5):E483-E491.

[9] Kobayashi T, Arai Y, Takeuchi Y, et al. Phase I/II clinical study of percutaneous vertebroplasty (PVP) as palliation for painful malignant vertebral compression fractures (PMVCF): JIVROSG-0202[J]. Ann Oncol,2009,20(12):1943-7.

[10] Martinez-Ferrer A, Blasco J, Carrasco J L, et al. Risk factors for the development of vertebral fractures after percutaneous vertebroplasty[J]. J Bone Miner Res,2013,28(8):1821-9.

[11] Nieuwenhuijse M J, Putter H, van Erkel A R, et al. New vertebral fractures after 
percutaneous vertebroplasty for painful osteoporotic vertebral compression fractures:

a clustered analysis and the relevance of intradiskal cement leakage[J].

Radiology,2013,266(3):862-70.

[12] Zhong B Y, He S C, Zhu H D, et al. Nomogram for Predicting Intradiscal Cement Leakage Following Percutaneous Vertebroplasty in Patients with Osteoporotic Related Vertebral Compression Fractures[J]. Pain Physician,2017,20(4):E513-E520.

[13] Zhang T Y, Zhang P X, Xue F, et al. Risk factors for cement leakage and nomogram for predicting the intradiscal cement leakage after the vertebra augmented surgery[J]. BMC Musculoskelet Disord,2020,21(1):792.

[14] Karakousis G, Sondak V K, Zager J S. Overestimation of Risk for Sentinel Lymph Node Metastasis in a Nomogram for T1 Melanomas[J]. J Clin Oncol,2020,38(27):3234-3235.

[15] Haas F, Byrne N M, Rey M. Nomogram for exercise capacity in women[J]. N Engl J Med,2005,353(21):2301-3; author reply 2301-3.

[16] Kao W Y, Su C W, Chiou Y Y, et al. Hepatocellular Carcinoma: Nomograms Based on the Albumin-Bilirubin Grade to Assess the Outcomes of Radiofrequency Ablation[J]. Radiology,2017,285(2):670-680.

[17] Zhang Z, Jing Q, Qiao R, et al. [Risk factors analysis of adjacent fractures after percutaneous vertebroplasty for osteoporotic vertebral compression fracture $][\mathrm{J}]$. Zhongguo Xiu Fu Chong Jian Wai Ke Za Zhi,2021,35(1):20-25.

[18] Kim H J, Zuckerman S L, Cerpa M, et al. Incidence and Risk Factors for Complications and Mortality After Vertebroplasty or Kyphoplasty in the Osteoporotic Vertebral Compression Fracture-Analysis of 1,932 Cases From the American College of Surgeons National Surgical Quality Improvement[J]. Global Spine $\mathrm{J}, 2020: 2192568220976355$.

[19] Bayram S, Akgul T, Adiyaman A E, et al. Effect of Sarcopenia on Mortality after Percutaneous Vertebral Augmentation Treatment for Osteoporotic Vertebral Compression Fractures in Elderly Patients: A Retrospective Cohort Study[J]. World Neurosurg,2020,138:e354-e360.

[20] Crouser N, Malik A T, Jain N, et al. Discharge to Inpatient Care Facility After 
Vertebroplasty/Kyphoplasty: Incidence, Risk Factors, and Postdischarge Outcomes[J]. World Neurosurg,2018,118:e483-e488.

[21] Long Y, Yi W, Yang D. Advances in Vertebral Augmentation Systems for Osteoporotic Vertebral Compression Fractures[J]. Pain Res Manag,2020,2020:3947368.

[22] Zhang Z, Jing Q, Qiao R, et al. [Risk factors analysis of adjacent fractures after percutaneous vertebroplasty for osteoporotic vertebral compression fracture $][\mathrm{J}]$. Zhongguo Xiu Fu Chong Jian Wai Ke Za Zhi,2021,35(1):20-25.

[23] Gupta A, Cha T, Schwab J, et al. Age Is Just a Number: Patient Age Does Not Affect Outcome Following Surgery for Osteoporotic Vertebral Compression Fractures[J]. Global Spine J,2020:2192568220941451.

[24] Kanis J A, Johansson H, Harvey N C, et al. The effect on subsequent fracture risk of age, sex, and prior fracture site by recency of prior fracture[J]. Osteoporos Int,2021.

[25] Bergh C, Wennergren D, Moller M, et al. Fracture incidence in adults in relation to age and gender: A study of 27,169 fractures in the Swedish Fracture Register in a well-defined catchment area[J]. Plos One,2020,15(12):e0244291.

[26] Cosman F. Anabolic Therapy and Optimal Treatment Sequences for Patients With Osteoporosis at High Risk for Fracture[J]. Endocr Pract,2020,26(7):777-786. [27] Osaki M, Okuda R, Saeki Y, et al. Efficiency of coordinator-based osteoporosis intervention in fragility fracture patients: a prospective randomized trial[J]. Osteoporos Int,2021.

[28] Bawa H S, Weick J, Dirschl D R. Anti-Osteoporotic Therapy After Fragility Fracture Lowers Rate of Subsequent Fracture: Analysis of a Large Population Sample[J]. J Bone Joint Surg Am,2015,97(19):1555-62.

[29] Kwon H M, Lee S P, Baek J W, et al. Appropriate Cement Volume in Vertebroplasty: A Multivariate Analysis with Short-Term Follow-Up[J]. Korean J Neurotrauma,2016,12(2):128-134.

[30] Fu Z, Hu X, Wu Y, et al. Is There a Dose-Response Relationship of Cement Volume With Cement Leakage and Pain Relief After Vertebroplasty?[J]. Dose 
Response,2016,14(4):1559325816682867.

[31] Roder C, Boszczyk B, Perler G, et al. Cement volume is the most important modifiable predictor for pain relief in BKP: results from SWISSspine, a nationwide registry[J]. Eur Spine J,2013,22(10):2241-8.

[32] Zhu S Y, Zhong Z M, Wu Q, et al. Risk factors for bone cement leakage in percutaneous vertebroplasty: a retrospective study of four hundred and eighty five patients[J]. Int Orthop,2016,40(6):1205-10.

[33] Chevalier Y, Pahr D, Charlebois M, et al. Cement distribution, volume, and compliance in vertebroplasty: some answers from an anatomy-based nonlinear finite element study[J]. Spine (Phila Pa 1976),2008,33(16):1722-30.

[34] Xie Hua, Li Jichun, He Jin, Xu Zhonghua, Nong Luming. Study on the effect of the distribution of bone cement on the efficacy of vertebroplasty. Chin J Orthop $[\mathrm{J}]$. 2017,37 (22): 1400-1406. 


\section{Figures}

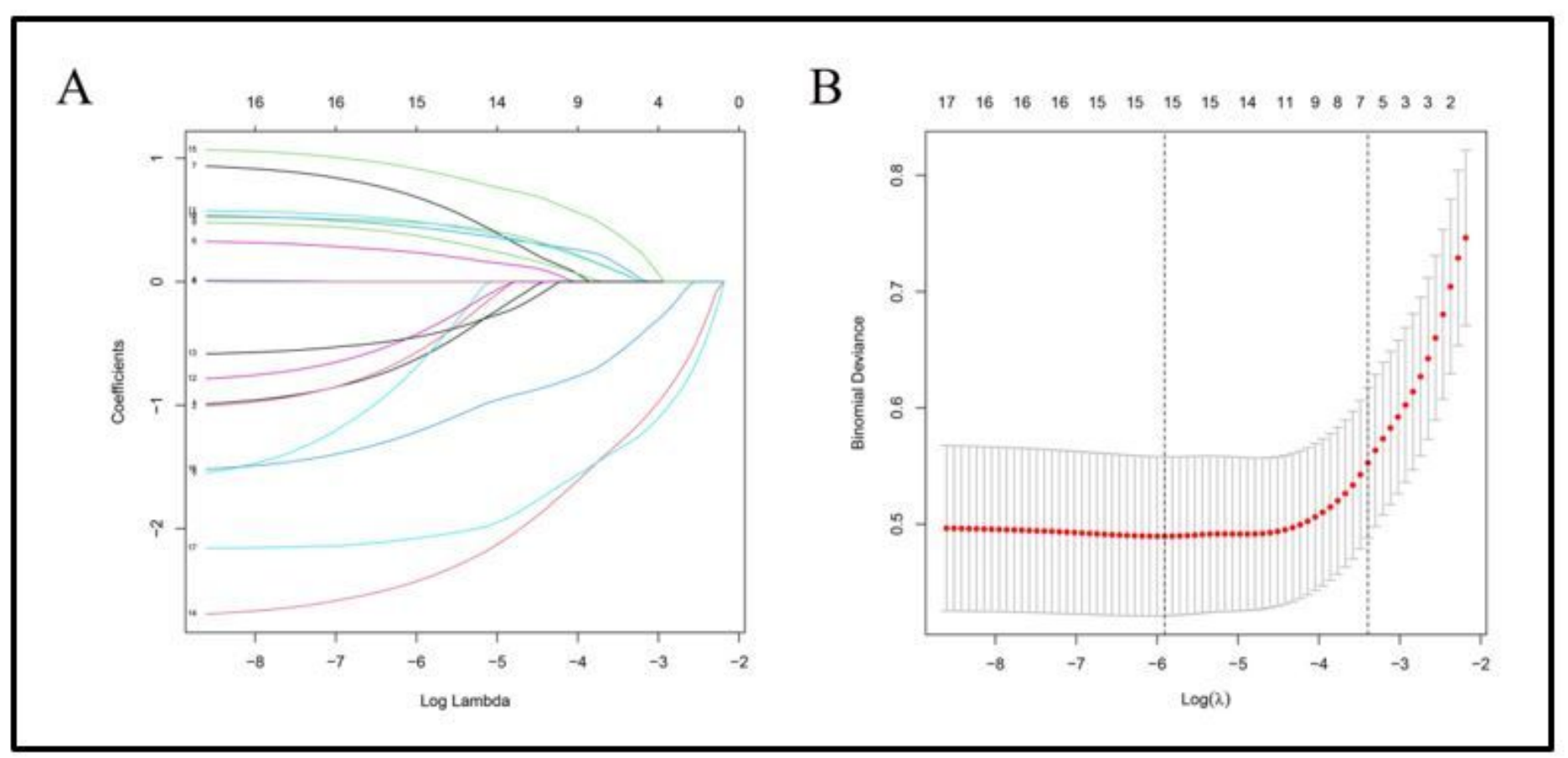

\section{Figure 1}

Demographic and clinical feature selection using LASSO binary logistic regression model. Note: 17 features of A cable coefficient profile. According to the logarithmic $(\lambda)$ sequence to draw the coefficient distribution map. Five-fold cross-validation is used to draw vertical lines at the selected values, where the best $\lambda$ results in six features with non-zero coefficients.The best parameter $(\lambda)$ in the $B$ lasso model is selected through the minimum criterion using five-fold cross validation. The relationship between the partial likelihood deviation (binomial deviation) curve and the logarithm $(\lambda)$ is drawn. Abbreviations: LASSO, minimum absolute shrinkage, selection operator; SE, standard error. 


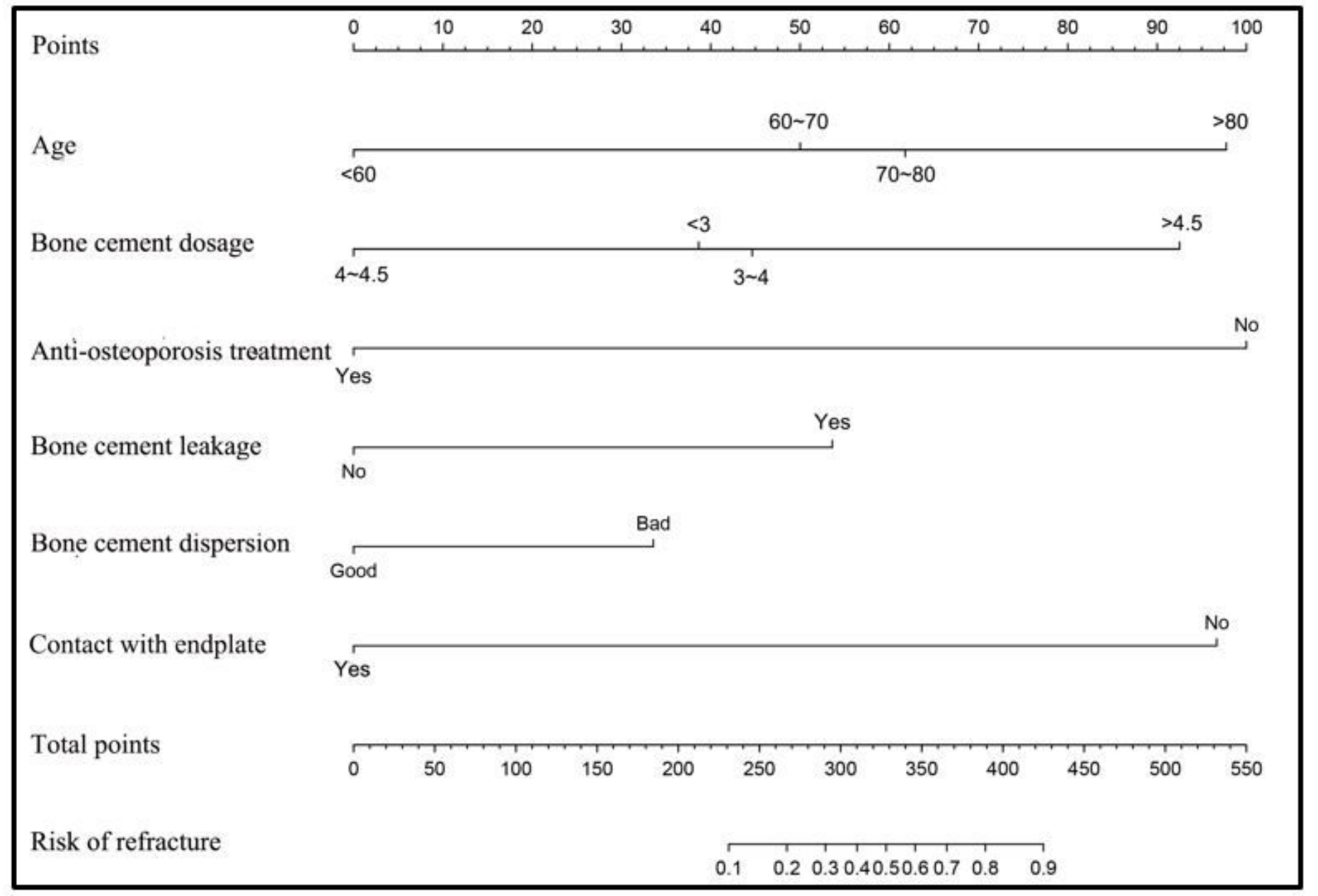

\section{Figure 2}

The developed nomogram of adjacent segmental fractures after vertebral augmentation. Note: The nomogram of postoperative vertebral compression fractures was developed in the cohort, including age, bone cement injection volume, bone cement leakage, bone cement dispersion, contact between bone cement and endplate, and available anti囚osteoporotic treatment. 


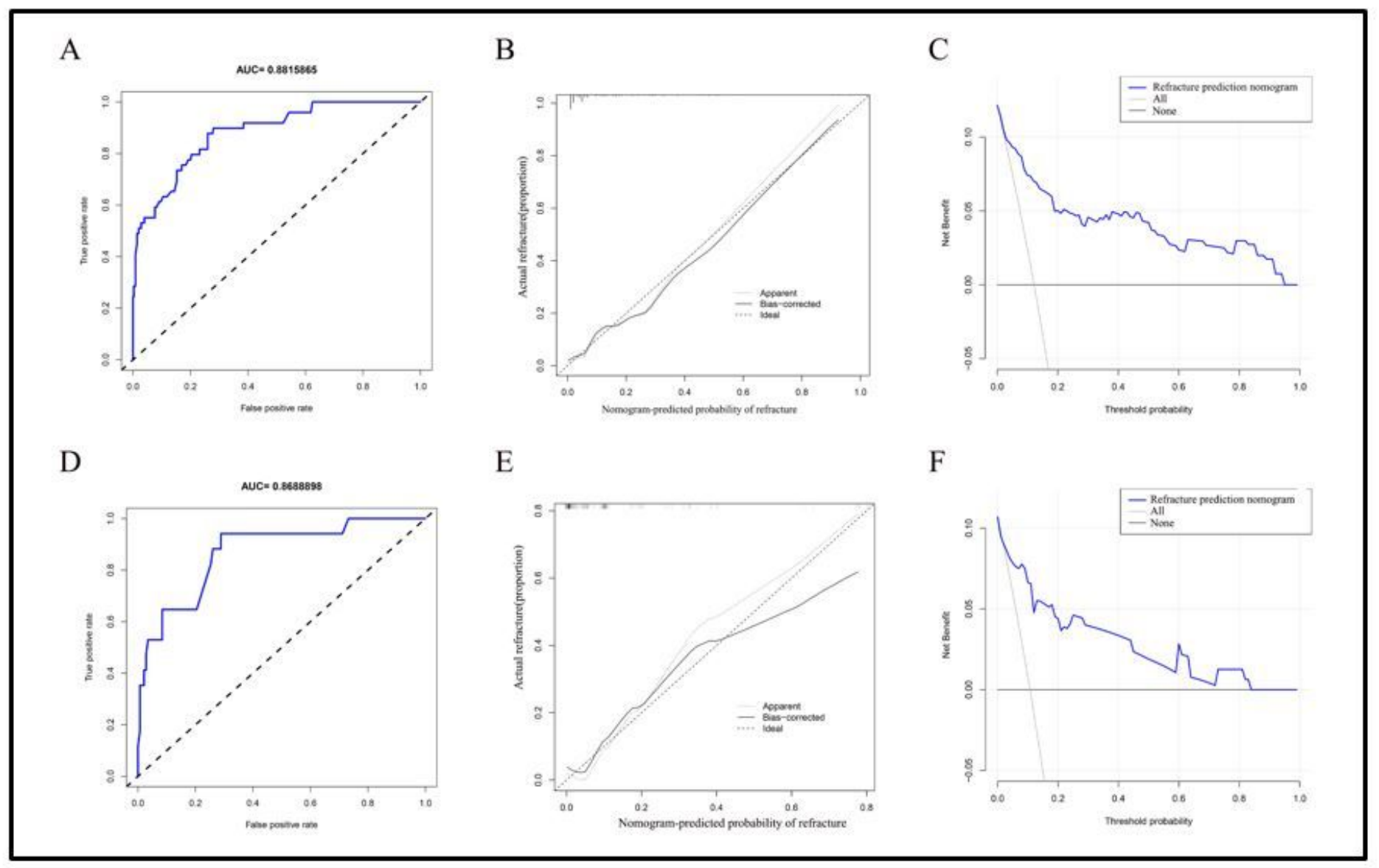

Figure 3

The receiver operating characteristic curve (A), calibration curve (B) and decision curve analysis (C) of the training cohort. Receiver operating characteristic curve (D), calibration curve (E) and decision curve analysis $(F)$ of the validation cohort. 